\title{
Buried waveguides in Nd:YLF crystals obtained by femtosecond laser writing under double line approach
}

\author{
D. Biasetti · E. Neyra • J.R. Vázquez de Aldana • \\ L. Roso • G.A. Torchia
}

Received: 11 August 2011 / Accepted: 3 August 2012 / Published online: 31 August 2012

(C) Springer-Verlag 2012

\begin{abstract}
In this paper, we present buried waveguides fabricated by fs laser writing in $\mathrm{Nd}^{3+}$ doped YLF crystal under double line approach (Miura et al. in Appl. Phys. Lett. 71:3329-3331, 1997). The waveguides were made by focusing two consecutive optical breakdown tracks (OBT) separated by about $20 \mu \mathrm{m}$. To make the optimal OBT, we focused the fs-laser pulses $200 \mu \mathrm{m}$ below surface at intensities above the OB threshold for the material and controlled the writing speed. The guiding structures were fabricated by using a Chirped Pulse Amplification (CPA) femtosecond (fs) laser system. We chose the optimal writing parameters in order to obtain suitable waveguides, using around $3 \mu \mathrm{J}$ energy and writing speed from 15 to $50 \mu \mathrm{m} / \mathrm{s}$. After optically exploring the waveguides by end-fire coupling, the guiding structures showed good optical performance. Guiding index profiles were retrieved from modal analysis by using BeamProp (RSoft) commercial software. This spatial distribution of the index increment, taking into account a lower refractive barrier on the OBT region plus the compressed region between the tracks, was obtained correctly fitting profiles modes.

Finally, optical spectroscopy measurements were also performed in the waveguides. The results showed that the luminescence properties of $\mathrm{Nd}^{3+}$ ions are preserved in the waveguides compared with the values obtained for bulk.
\end{abstract}

D. Biasetti · E. Neyra · G.A. Torchia $(\bowtie)$

Centro de Investigaciones Ópticas CONICET La Plata-CIC, Camino Centenario y 506, MB Gonnet (1897), Pcia. Bs. As., Argentina

e-mail: gustavot@ciop.unlp.edu.ar

J.R. Vázquez de Aldana $\cdot$ L. Roso

CLPU and Servicio Láser de la Universidad de Salamanca, Plaza

de la Merced s/n 35008 Salamanca, Spain

\section{Introduction}

From the appearance of the Chirped Pulse Amplification femtosecond (fs) systems, the interaction of this kind of laser pulses with optical materials gives rise to an important research field which involves the fs laser writing interaction with optical materials. As it is well known, by using this technology, nonlinear processes are the main responsible for the material processing, thus it is possible to modify, in a controlled way, any optical materials by using this experimental procedure. This modification is firstly activated by the ionization process which can be reached via multiphoton absorption, avalanche or tunnel. After this process, optical modification, damage or breakdown can be achieved in the material, depending on the laser fluences used. During this process, hugely free electrons within the plasma promoted by the laser interaction are recombined in the network, so, the fast cooling and shock waves originated are the responsible for the formation of high density regions, residual stress, defects, amorphous zones, etc. in the material $[1,2]$.

In this sense, nowadays, the femtosecond (fs) laser writing represents a well established technique to achieve optical circuits in a large number of materials by using either optically passive or active samples [2-8]. As it is well known, the technique can be conducted in any material by adjusting the experimental parameters to obtain the desired guiding structure by means of different writing strategies. In this work we fabricated the buried waveguides by the socalled "double line approach" method; this means that the guiding structure is defined between two parallel and very close $(15 \mu \mathrm{m})$ optical breakdown tracks (OBT) which were generated by laser writing. As it has been reported in several papers, this interaction region is achieved at intensities above the optical breakdown threshold where Coulomb explosion takes place inside the material so that the interaction 
regions keep a residual stress surrounding the tracks (guiding region). Besides, the OBT larger expansion takes places, therefore lower density walls also support the confined light in these guiding structures. This kind of optical waveguides guarantees high power confinement as well as the possibility of tailoring several closed regions to guide the light even and the best advantage is that they fabricate 3D optical guiding structures [9-15].

The aim of this work was to achieve integrated waveguides in short phonon systems in order to improve the luminescence efficiency; the fluorides form part of this kind of materials [23, p. 209]. These materials are important for efficient luminescence applications since the luminescence quantum efficiency is large due to the lower probability to couple a large number of low energy phonon against the radiative de-excitation. Besides, as it is well known, the YLF crystal has a good thermal conductivity so that some drawback effects such as induced thermal birefringence and lensing can be negligible $[16,17]$. On the other hand, the $\mathrm{Nd}^{3+}$ ions as luminescence centers represent a synonym to the laser excellence for any doping optical system as it has been reported during the last decades [18, 19].

From a luminescence point of view, also the $\mathrm{Nd}^{3+}$ within YLF crystal has advantages since it can be pumped for longtime sources as diode lasers because of larger luminescence lifetime in $\mathrm{Nd}: \mathrm{YLiF}_{4}$ which is twice longer than in Nd:YAG crystal.

In this paper we present suitable waveguides fabricated by femtosecond laser writing under the double line approach in $\mathrm{Nd}^{3+}$ doped YLF crystals. In this sense, propagation performance, as well as optical spectroscopy, analyses were carried out. Also, by using commercial software we obtained the refractive index profile for the waveguide fabrication procedure used in this work.

\section{Experimental procedure}

The waveguides were conducted following the well-established technique of laser direct writing under double line approach [20], i.e., these guiding structures were made by focusing a CPA Ti:Saphire laser beam (centered at a wavelength of $796 \mathrm{~nm}$ ) into a rectangular sample of $10 \mathrm{~mm}(\mathrm{~b}) \times$ $16 \mathrm{~mm}$ (c) and $2 \mathrm{~mm}$ (a) thickness of Nd:YLF (YLiF 4$)$ crystal keeping the relative displacement for both tracks written at the same velocity, being the translation perpendicular to the incident beam (transversal geometry). Using a repetition rate of the CPA system of $1 \mathrm{kHz}$ with about $120 \mathrm{fs}$ duration per pulse, the beam was focused $250 \mu \mathrm{m}$ below sample surface. As the result of the interaction is mainly determined by two parameters, the laser fluence and the writing speed, we manipulated these to obtain different OB tracks quali-

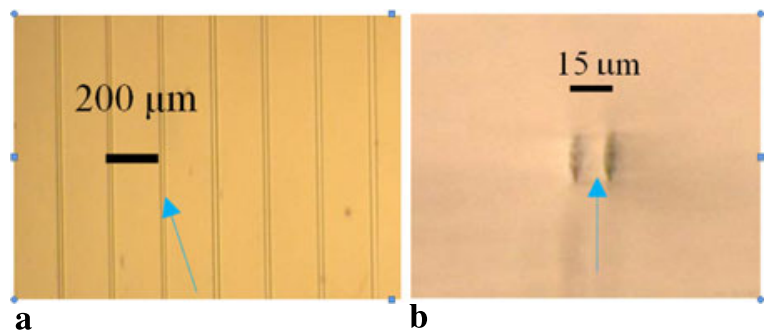

Fig. 1 (a) The picture shows a top view from the waveguide fabricated in this work (left). The arrow is pointed to the waveguide which was investigated in this work. (b) Optical microscopy image of a cross section corresponding to a waveguide is detailed

ties. The waveguides were fabricated along the $\mathrm{c}$ axis ${ }^{1}$ of the crystal. Previously to the beam focused by Leica lens of $20 \times$, the energy per pulse was set at 30 and $50 \mu \mathrm{J}$ thus corresponding to a fluence of 25 and $42 \mathrm{~J} / \mathrm{cm}^{2}$ in the sample, taking into account the attenuation due filtering and losses by an estimated factor of $6 \times 10^{-2}$ for our arrangement. The sample was moved at a speed ranging from 15 to $50 \mu \mathrm{m} / \mathrm{s}$ by using a motorized translation stage in a micromachining station which is detailed in [14]. For generating the double tracks we considered three different separations between consecutive straight lines, 15,20 and $25 \mu \mathrm{m}$.

\section{Results and discussion}

\subsection{Waveguides obtained}

In Fig. 1(a) we can see the top view of the guiding structures obtained in this work. The lines show an almost smooth track so we can expect good optical performance from these guiding structures. On the other hand, Fig. 1(b) presents the cross section from one of the buried waveguides fabricated in this work. As it can be seen. the black tracks correspond to the focused laser region and it is well known that the brilliant region between them corresponds to the guiding zone, pointed by an arrow in the figure.

\subsection{Optical propagation performance}

In order to test light modes propagation in the visible generated tracks shown in Fig. 1, we assembled an end-fire system coupling as it is described in Ref. [21]. Focusing with a $10 \times$ objective laser beam peaked at $532 \mathrm{~nm}$ in one of the polished faces of the sample where the waveguides are located parallel to laser propagation. At the output a similar $10 \times$ microscope objective was used to collect the near field from the waveguide. By focusing the output mode on the beam

\footnotetext{
${ }^{1}$ We chose this axis because of the lower absorption for the ordinary propagation.
} 
profile analyzer (BPA) it was possible to find the intensity modal distribution collecting the near-field pattern emerging from the end-face. Taking into account the lens magnification and the pixel resolution of BPA, the real size of modal distribution could be determined. Then, the optical quality of the waveguides was checked from the modal distribution of each one. As a result, all the waveguides showed good optical performance. Figure 2 shows the mode profile corresponding to typical waveguide fabricated in this paper.

Finally, the propagation losses were estimated by means of measuring the scattered light from the top of the waveguides, which was recorded in a picture. By averaging many registered pictures for the same waveguides we obtained a value of $2 \mathrm{~dB} / \mathrm{cm}$, corresponding to the propagation losses for the above guiding structure.

\subsection{Simulation of index refractive profiles}

By using commercial simulation software RSoft, it was possible to simulate a double track waveguide, performed over Nd-YLF crystal. The design tool BeamProp used was based of the BPM (Beam Propagation Method) technique. This well-known numerical method is used to solve the Helmholtz equation of a monochromatic wave.

The BMP method supposes that the variation of the wave shape is small over the propagation axis. Thus, using the Crank-Nicholson method of finite elements and imposing transparent boundary conditions allowed solving the monochromatic wave equation. To perform this simulation,

Fig. 2 Typical near-field profile of the waveguide fabricated in this work with a launched wavelength of $532 \mathrm{~nm}$. Dashed line box is just automatically inserted by the commercial software of CCD used to obtain the profile, enclosing the zone with higher intensity
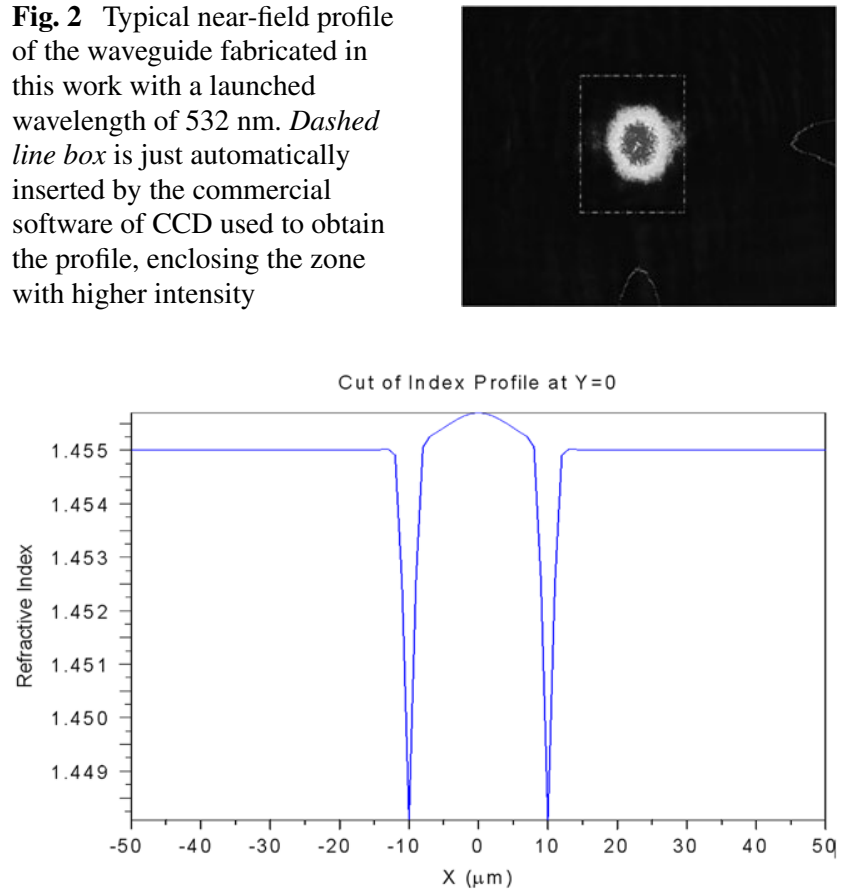

Fig. 3 Cross section of function plot describing the refractive index profile used to start the simulation with the RSoft software a profile of refractive index was made as we assumed a starting point for fitting a sum of Gaussian functions [9] as it is sketched in Fig. 3. This index profile consisted of a central Gaussian function, which represented an increment about $10^{-3}$ of the refraction index, and two symmetric Gaussian functions with index of about $-10^{-2}$. The distance between the lateral Gaussian functions was set at $20 \mu \mathrm{m}$, similarly to those used for the fabricated waveguides.

Based on a picture for the mode profile obtained from a beam profile analyzer (BPA), and comparing it with the mode given by the simulation, on the suggested Gaussian profile it was possible to adjust the different index increments until making coincide the simulated and the real mode. In this way we were able to retrieve the variation of the refractive index obtained by this waveguide fabrication procedure.

To perform the fitting of these modes, in the first place an increment of the refraction index profile of $10^{-3}$ and a drop of $10^{-2}$ were suggested (see Fig. 4). Then the simulation was performed and the resulting mode was compared with the mode obtained by the BPA; since these modes did not fit, the increment of the refraction index was adjusted until the simulated mode fitting with that obtained with the beam profile analyzer (BPA). This was done by measuring the real dimensions of the mode recorded by BPA and taking into account the zoom given by the optical system and knowing the CCD dimensions.

The final index profile adjust is shown in Fig. 5. The wavelength used in the simulation was $532 \mathrm{~nm}$, which is the wavelength used to propagate in the optical experiments. Also in Fig. 5, two modes, one corresponding to the simulation and the other to the mode taken with the BPA for a waveguide fabricated with $2 \mu \mathrm{J}$ and $25 \mu \mathrm{m} / \mathrm{s}$, are shown.

Finally, we also performed a simulation for a wavelength of $1064 \mathrm{~nm}$ in order to know the available laser performance for these waveguides. The refraction index profile used was that obtained after the above fitting (Fig. 5). The mode given

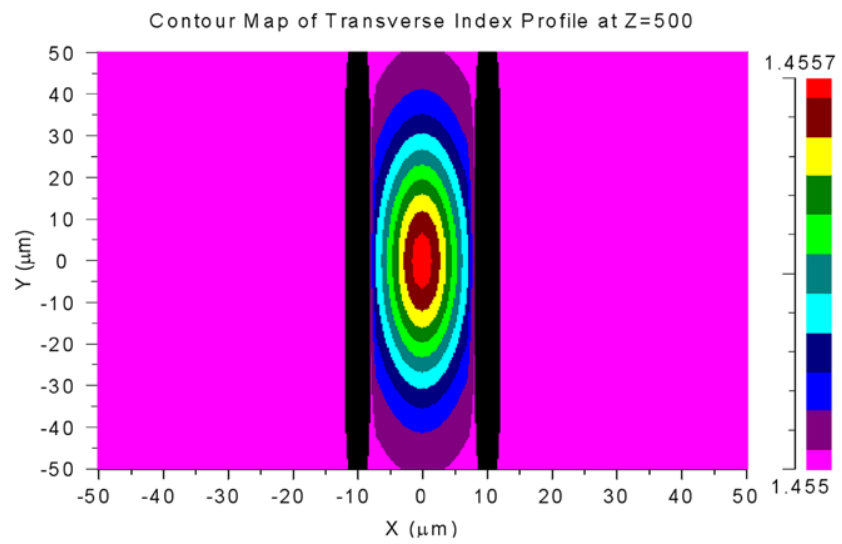

Fig. 4 Refractive index profile in the crystal resulting after fitting the mode profile as is described in the text 


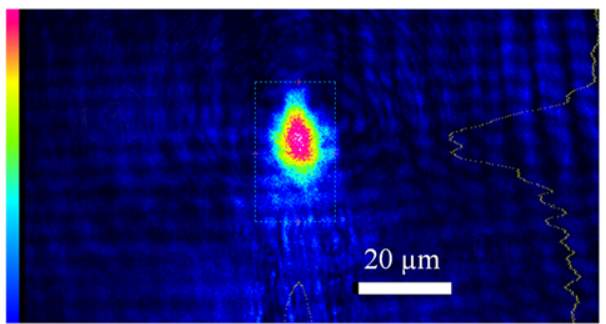

a
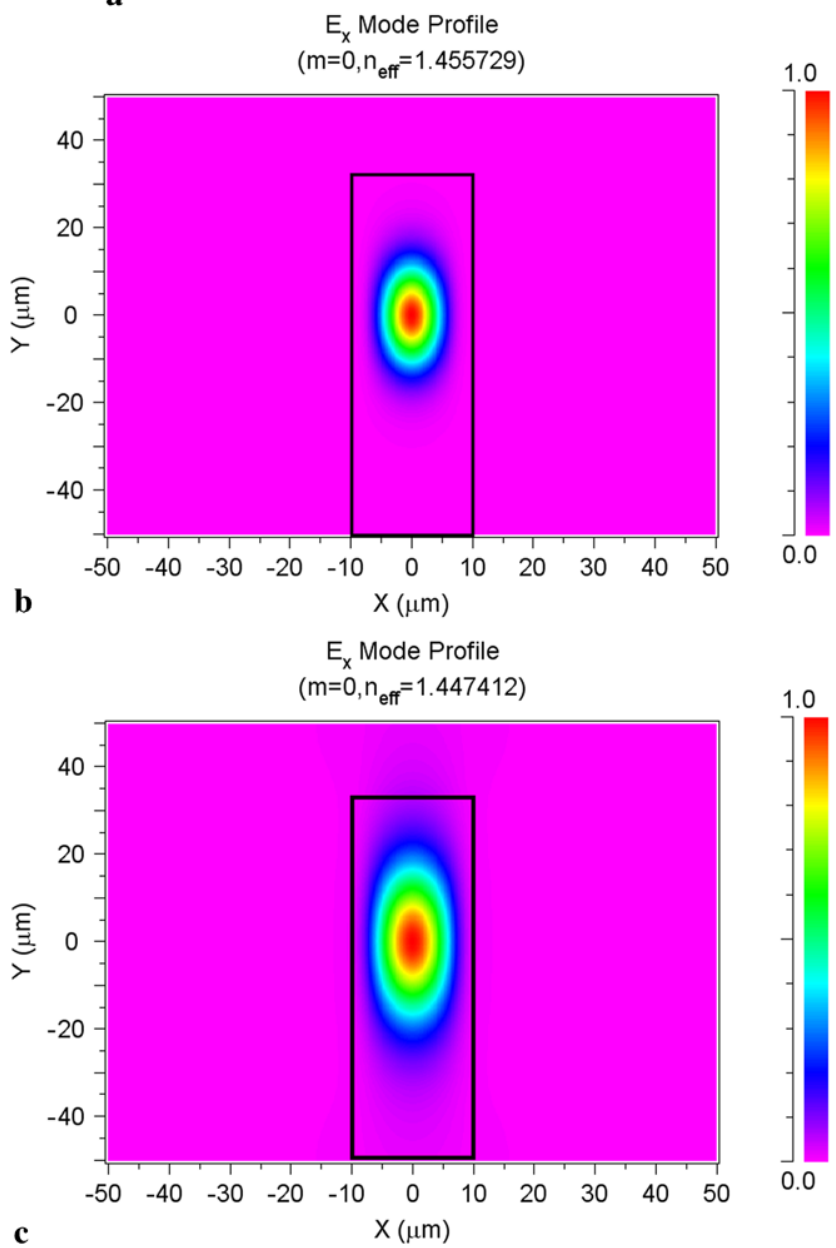

Fig. 5 Experimental (a) and simulated (b) guided mode propagated at $532 \mathrm{~nm}$ inside waveguide are shown. Also, simulated mode at $1064 \mathrm{~nm}$ obtained from the resulted guiding structure is shown in (c). In (b) and (c) the inset box shows waveguide's region

by this simulation is also shown in Fig. 5(c). As can be seen, the mode is almost confined within the waveguide region which is delimited by an open black box.

\subsection{Optical spectroscopy measurements}

Two goals were achieved concerning optical spectroscopy properties of $\mathrm{Nd}^{+3}$ as dopant of YLF crystal. The first one was to compare bulk and waveguide mode photoluminescence shape spectrum, and the second to estimate lifetime of photoluminescence $\mathrm{Nd}^{+3}$ laser level ${ }^{4} \mathrm{~F}_{3 / 2}$. Whereas

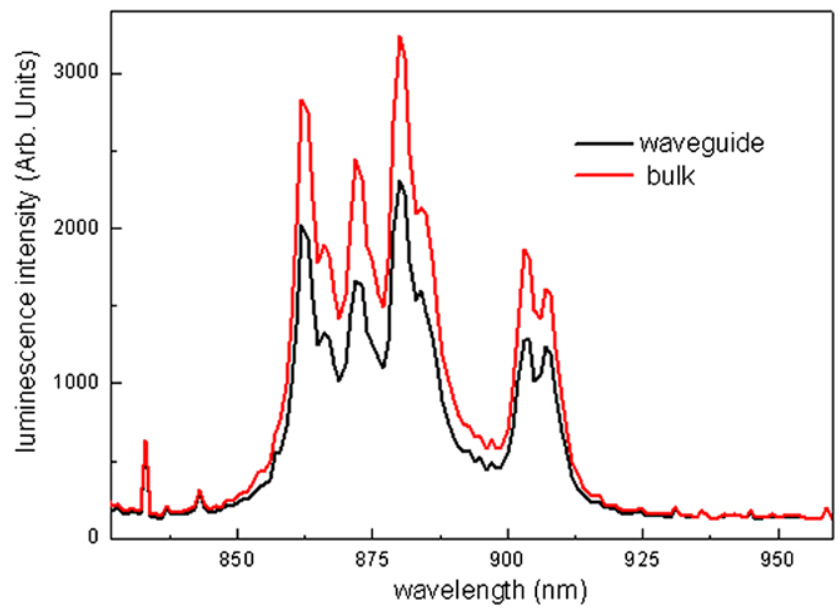

Fig. 6 Luminescence spectra after laser excitation at $532 \mathrm{~nm}$. The red and black lines correspond to waveguide and bulk emission spectra

for the former we worked in the continuous wave $(\mathrm{cw})$ regime, the latter was reached working under pulsed excitation laser using a mechanical chopper. For measurements, an optical cut filter was used to suppress the pump wavelength.

The experimental arrangement was similar to that used for optical performance measurements in Ref. [22]. The output luminescence after pumping with $\mathrm{cw}$ laser at $532 \mathrm{~nm}$ was focused into a fiber optical pigtail spectrometer.

We explored the IR emission spectra, corresponding to ${ }^{4} \mathrm{~F}_{3 / 2} \rightarrow{ }^{4} \mathrm{I}_{9 / 2}$ transition (from 850 to $920 \mathrm{~nm}$ ). This occurs after non-radiative connection from ${ }^{2} \mathrm{G}_{9 / 2}$ and ${ }^{4} \mathrm{G}_{11 / 2}$ manifold to meta-stable ${ }^{4} \mathrm{~F}_{3 / 2}$ level. In Fig. 6 this luminescence transition is observed including several peaks due to Stark levels degeneracy [23]. Comparing the spectra, it can be clearly observed that the shape spectrum corresponding to $\mathrm{Nd}^{+3}$ ions is similar for the waveguide and bulk. This is related to the fact that the waveguide fabrication process keeps the main optical properties in the waveguides as in bulk.

On the other hand, in order to obtain lifetime of ${ }^{4} \mathrm{~F}_{3 / 2}$, the luminescence output signal from the waveguide was focused onto a silicon detector connected to a digital oscilloscope. Fitting the logarithmic of exponential decay temporal signal, we obtained the constant which is the reciprocal lifetime, as slope reciprocal. The results are presented as the fit slope of linear curve in Fig. 7 for both bulk and waveguide emission, being the slopes perfectly matched within experimental error. Both lifetime decays are ranged around $480 \pm 20 \mu$ s. As we expected, the waveguides fabrication technique preserved the spectroscopic characteristic of $\mathrm{Nd}^{3+}$ ions as they represent a good candidate to perform laser action in this kind of waveguide configuration. 


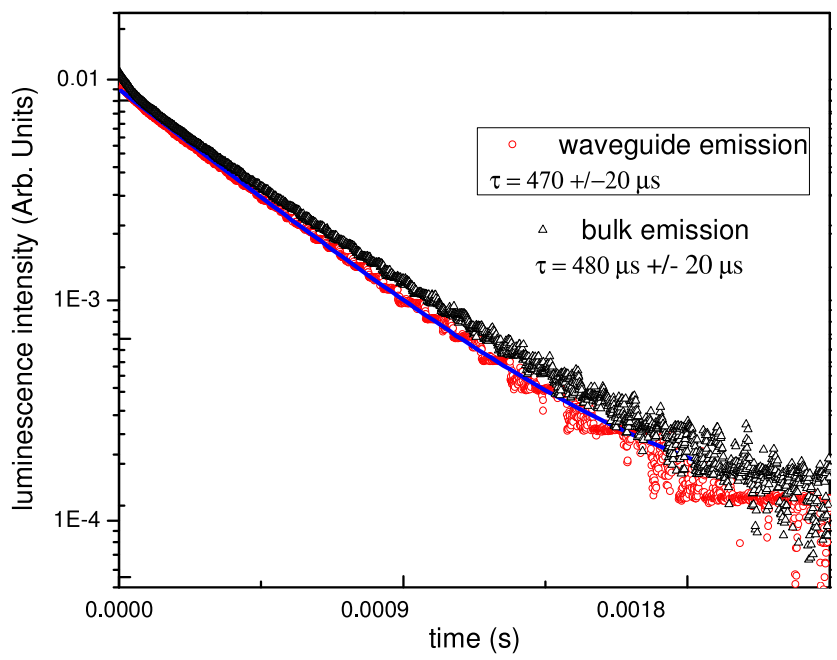

Fig. 7 Lifetime decays for the luminescence coming from the ${ }^{3} \mathrm{~F}_{3 / 2}$ meta-stable level corresponding to the $\mathrm{Nd}^{3+}$ ions in YLF crystals. The open circles and triangles correspond to the waveguide and bulk

\section{Conclusions}

In this work we have presented suitable buried waveguides made in $\mathrm{Nd}^{3+}$ doped YLF crystals under double line approach by means of femtosecond laser writing. A propagation loss of $2 \mathrm{~dB} / \mathrm{cm}$ has been determined by registering the scattering light from the top of the guiding structures.

By using commercial software RSoft the index profile modification has been determined. For this, fitting the size of the output and the simulated mode has been the key to obtain the refractive index structure. The laser tracks have a decrement of refractive index of about $10^{-2}$ while the region between tracks reported an index increment of about $10^{-3}$.

Finally, from the laser excitation spectroscopic test the luminescence properties corresponding to $\mathrm{Nd}^{3+}$ ions have been preserved in these guiding structures as it has been checked from the luminescence spectra and the higher measured lifetime $(480 \mu \mathrm{s})$.

From the results found in this paper, the buried waveguides in $\mathrm{Nd}^{3+}$ doped YLF crystals fabricated by fs laser writing are a good candidate for laser action with best predictions.

Acknowledgements This work was partially supported by Agencia Nacional de Promoción Científica y Tecnólogica. (Argentina) under project PICT-2575 and CONICET (Argentina) under project PIP 0394 and by "Ministerio de Educación y Ciencia," Spain (Grant $\mathrm{n}^{\circ}$ FIS2009-09522 and Consolider Program SAUUL CSD2007-00013). D.B. wishes to thank Comisión de Investigaciones Científicas (Bs. As., Argentina) for his student fellowship.

\section{References}

1. J. Perriere, E. Millon, E. Fogarassy (eds.), Recent Advances in Laser Processing of Materials. European Materials Research Society Series (2006)

2. E. Gamaly, Femtosecond Laser Matter Interactions: Theory, Experiments and Applications (Pan Stanford Publishing Pte. Ltd., Singapore, 2011)

3. K. Miura, J. Qui, H. Inouye, T. Mitsuyu, K. Hirao, Appl. Phys. Lett. 71, 3329-3331 (1997)

4. E.N. Glezer, E. Mazur, Appl. Phys. Lett. 71, 882 (1997)

5. A.M. Strelsov, N.F. Borrelli, J. Opt. Soc. Am. B 19, 2496-2504 (2002)

6. M. Will, S. Nolte, B.N. Chichkov, A. Tunnermann, Appl. Opt. 41, 4360-4364 (2002)

7. C. Florea, K.A. Winick, Fabrication and characterization of photonic devices directly written in glass using femtosecond laser pulses. J. Lightwave Technol. 21, 246-253 (2003)

8. S. Nolte, M. Will, J. Burghoff, A. Tunnermann, Appl. Phys. A 77, 109-111 (2003)

9. S. Taccheo, G. Della Valle, R. Osellame, G. Cerullo, N. Chiodo, P. Laporta, O. Svelto, A. Killi, U. Morgner, M. Lederer, D. Kopf, Opt. Lett. 29, 1900-1902 (2004)

10. A.G. Okhrimchuk, A.V. Shestakov, I. Khrushchev, J. Mitchell, Opt. Lett. 30, 2248-2250 (2005)

11. J. Burghoff, H. Hartung, S. Nolte, A. Tunnermann, Appl. Phys. A 86, 165-170 (2006)

12. N.T. Nguyen, A. Saliminia, S.L. Chin, R. Vallée, Appl. Phys. B 85, 145-148 (2006)

13. G. Della Valle, S. Taccheo, R. Osellame, A. Festa, G. Cerullo, P. Laporta, Opt. Express 15(6), 3190-3194 (2007)

14. C. Méndez, J.R. Vázquez de Aldana, G.A. Torchia, L. Roso, Arrays of guiding structures induced in fused silica by void like defects using femtosecond laser pulses. Appl. Phys. B, Laser Opt. 86, 343-346 (2007)

15. G.A. Torchia, A. Ródenas, A. Benayas, L. Roso, D. Jaque, Highly efficient laser action in femtosecond-written $\mathrm{Nd}$ :yttrium aluminum crystals. Appl. Phys. Lett. 92, 111103 (2008)

16. H. Vanherzeele, Thermal lensing measurement and compensation in a continuous-wave mode-locked Nd:YLF laser. Opt. Lett. 13, 369-371 (1988)

17. G. Cerullo, S.D. Silvestri, V. Magni, High efficiency, $40 \mathrm{~W} \mathrm{cw}$ nd: YLF laser with large TEM00 mode. Opt. Commun. 93, 77-81 (1992)

18. A.A. Kaminskii, Laser Crystals (Springer, New York, 1981)

19. W. Koechner, Solid-State Laser Engineering, 6th edn. (Springer, Berlin, 2006)

20. A. Rodenas, G.A. Torchia, G. Lifante, E. Cantelar, F. Jaque, D. Jaque, L. Roso, Appl. Phys. B 95, 85-96 (2009)

21. E. Cantelar, D. Jaque, G. Lifante, Waveguide lasers based on dielectric materials. Opt. Mater. 34, 555-571 (2012)

22. G.A. Torchia, C. Méndez, L. Roso, J.O. Tocho, J. Lumin. 128, 754-756 (2008)

23. J. Garcia Sole, D. Jaque, L.E. Bausa, An Introduction to the Optical Spectroscopy of Inorganic Solids (Wiley, New York, 2005) 